\title{
China Can Substantially Reduce Its High Burden of Stroke and Heart Attack
}

\author{
Thomas R Frieden ${ }^{*}$
}

Hypertension, the leading cause of cardiovascular disease, kills 10.7 million people worldwide each year - more than any other cause, and more than all infectious diseases combined (1). Approximately one third of adults globally have elevated blood pressure; of these approximately 1.4 billion people, only 1 in 7 with hypertension are effectively treated so that their blood pressure is reduced to below 140/90 (2).

China's hypertension burden mirrors the global situation. A quarter of a billion people in China have hypertension - the most of any country in the world - with $23.5 \%$ of the adult population having elevated blood pressure. Only an estimated 10\%-15\% of those with hypertension are treated effectively such that they have the condition under control (3).

Each year, 1.8 million people in China die from hypertension, nearly double the number in India despite similar population sizes and rates of hypertension (4). For a productive and healthy future, prevention and control of hypertension is essential. It is also feasible to do so; for example, Canada has improved hypertension control rates to nearly $70 \%$ nationwide (5), and Thailand's rate of blood pressure control increased more than 3-fold, from $8.6 \%$ to $30 \%$, between 2004 and 2014 (G), and appears to have continued to increase since.

The two most effective methods of reducing the strokes, heart attacks, and other health problems hypertension causes are to improve hypertension treatment and reduce the intake of sodium, which is a leading contributor to high blood pressure. Globally over the next 25 years, improving control of hypertension from the current $14 \%$ of people with high blood pressure to $50 \%$ would prevent about 40 million cardiovascular deaths, and reducing population sodium intake by $30 \%$ would prevent another 40 million deaths $(7)$.

Of all primary care interventions for adults, improvement in control of hypertension can save the most lives, potentially many times the number from many other interventions (8). Excess consumption of dietary sodium increases blood pressure and cardiovascular disease; the average sodium intake in
China is approximately double the recommendations of World Health Organization (WHO) and other leading health organizations (9). These two interventions will be integral to achieving Sustainable Development Goal 3.4.1, which aims to reduce the risk of premature death among people aged 30-69 years from noncommunicable diseases by one third by 2030 (10), as well as to achieve the aims of Healthy China 2030 (11).

Health systems that succeed in hypertension control ensure provision of a technical package with 5 key components: 1) protocols that establish standard treatment; 2) community-based care, including use of health staff at the community level to do more tasks such as blood pressure measurement, medication refills, and medication titration following a protocol, with or without telemedicine or artificial intelligence support; 3) regular and uninterrupted supply of quality medications and blood pressure monitoring equipment; 4) patient-centered services that reduce barriers to adherence including the complete elimination of fees for medical visits and for antihypertension medications; and 5) information systems that allow accurate and reliable real-time feedback on blood pressure control to clinical staff and program managers (12). These elements are also reflected in the WHO HEARTS technical package for cardiovascular disease management in primary health care (13).

From the perspective of hypertension control, four aspects of this technical package are particularly important in the Chinese context:

1. Adhering to specific, algorithmic treatment protocols to eliminate unwarranted variability in prescribing, and facilitate task-sharing and decentralization, and reduce health system and patient out-of-pocket costs while improving treatment efficacy. Reduced patient costs are demonstrated to increase adherence to treatment regimens and thereby reduce the financial and societal costs of avoidable heart attacks and strokes.

2. Providing three- or six-month refills with appropriate appointment spacing for stable patients, using virtual consultation and patient self-management when feasible. This will also reduce costs for the health 
system and for patients and increase patient retention in care. If China is to double, triple, or quadruple the proportion of patients with hypertension who have it under control, it will need to increase the efficiency of treatment - less frequent visits with longer refills are one important way to do this. These reforms, implemented during China's successful campaign to stop COVID-19 by limiting visits to health facilities, are excellent innovations and will benefit patients and the health system if they are made permanent throughout China.

3. Improving patient access to care by reducing reliance on hospital-centric care and increasing use of community-based primary care. China has committed to shifting its focus and resources toward safe, effective, accessible, and affordable basic health services at lower levels of care (14), and specifically adopting this more integrated approach by augmenting its primary care workforce (15). Making care easier to access will increase treatment adherence, reduce population hypertension rates, and save lives.

4. Developing a functional health information system with interoperability between health facilities and the ability to collect and analyze data on meaningful, consistent, reliable indicators of blood pressure control. This is essential to program evaluation and improvement.

Reducing dietary sodium intake and associated hypertension and cardiovascular disease is possible, as has been documented in the United Kingdom (UK) (16) and Finland (17). More recently, the Republic of Korea implemented a multicomponent program that reduced dietary sodium consumption among adults by $24 \%$ over a 4-year period, with reductions in population blood pressure and hypertension prevalence (18). Although there are differences in food consumption profiles, lessons from the Republic of Korea program are likely relevant to China and other Asian countries.

There are 3 primary sources of dietary sodium: 1) sodium added to packaged food during manufacturing; 2) sodium added to food prepared and consumed outside the home (e.g., restaurants, cafeterias, street food vendors); and 3) sodium added in the home, either during cooking or while eating (9). In China, two thirds of dietary sodium intake comes from these in-home sources (19); sodium reduction efforts will need to address this to be successful.

China already has a model to reduce sodium added in the home: the Shandong-Ministry of Health Action on Salt and Hypertension (SMASH) program. This intensive program used a mix of promotion of potassium-containing, low-sodium salt, community education, mass media, distribution of salt measuring spoons, and collaboration with restaurants and supermarkets to set food standards for sodium content. This intervention led to a reported $25 \%$ reduction in sodium intake as measured by 24-hour urine sodium and significant declines in blood pressure (20).

Urban populations are more likely to consume commercially packaged foods and foods prepared outside the home, and these sources of dietary sodium must also be addressed. Working with packaged food manufacturers to reduce sodium content has been successful in the UK, Republic of Korea, and other countries; mandatory reductions are much more effective than voluntary initiatives, which usually fail. Establishing specific targets to reduce sodium in different food categories nationwide, along with requirements to label sodium content, is a feasible approach.

In addition, front-of-package labeling highlighting levels of sodium, fats, and sugar help consumers quickly and effectively make healthier choices. In particular, mandatory front-of-package labels that feature warning signs, such as introduced in Chile, have been demonstrated to lead to significant reduction in purchases of sugar-sweetened beverages and are the emerging best practice globally for front-ofpack images (21). Chile's front-of-package warning labeling of high-salt and high-fat foods is likely to result in both improved options for consumers and improved choices by consumers. Sodium intake can also be reduced in foods prepared and eaten outside the home. For example, strong and mandatory policies which set standards for food that is procured or served on public property have been demonstrated to improve the availability of healthy food in institutions such as school and public workplaces (22).

Adopting a mix of proven interventions such as those demonstrated in SMASH mandatory policies supporting sodium targets, front-of-package labels, and public food procurement can lead to significant reductions in sodium intake, preventing hypertension and improving its control. However, additional innovative measures should also be investigated for use in China. These include increased use of low-sodium salt and other low-sodium condiments as well as interventions to reduce sodium use in restaurantprepared foods and to provide customers with information on key nutrients in restaurant menu items.

Although China has made some progress improving hypertension treatment and reducing dietary sodium intake, stronger action on both fronts can save millions of lives and billions of yuan. China has the opportunity to become a regional and global model for reducing 
hypertension prevalence and preventing cardiovascular disease, which causes nearly half of all deaths in the country each year (23). Millions of lives could be saved and much disability from stroke, heart attack, blindness, kidney failure, dementia, and other complications of hypertension could be prevented in China if the laudable Healthy China 2030 commitment to hypertension treatment and sodium reduction become realities in practice.

The fundamental emerging global insight on cardiovascular prevention is clear: the most important issue is economics. High and increasing taxes on tobacco and alcohol, controls on marketing and availability of tobacco and alcohol, much higher, capitated pay for primary care clinicians, a health care payment model that rewards providers substantially for validated hypertension control and eliminates costs for patients, and making healthy, low-sodium food less expensive than unhealthy food are the road not just to health, but also to productivity, economic stability, and societal progress.

\section{doi: $10.46234 / \mathrm{ccdcw} 2020.211$}

\# Corresponding author: Dr. Thomas R Frieden, tfrieden@rtsl.org. Thomas R Frieden (MD, MPH) is former director of the US Centers for Disease Control and Prevention, and former commissioner of the New York City Health Department. He is currently President and CEO of Resolve to Save Lives, an initiative of the global health organization Vital Strategies. Resolve works with countries to prevent 100 million deaths and make the world safer from epidemics. Vital Strategies is officially registered as an international non-governmental organization in China, with headquarters in Shandong Province.

Submitted: September 15, 2020; Accepted: September 22, 2020

\section{REFERENCES}

1. World Health Organization. Disease burden and mortality estimates: Cause-specific mortality, 2000-2016. Geneva: World Health Organization; 2018. https://www.who.int/healthinfo/global_burden_ disease/estimates/en.

2. Mills KT, Bundy JD, Kelly TN, Reed JE, Kearney PM, Reynolds K, et al. Global disparities of hypertension prevalence and control: a systematic analysis of population-based studies from 90 countries. Circulation 2016;134(6):441 - 50. http://dx.doi.org/10.1161/CIRCUL ATIONAHA.115.018912.

3. Wang ZW, Chen Z, Zhang LF, Wang X, Hao G, Zhang ZG, et al. Status of hypertension in China: results from the China Hypertension Survey, 2012-2015. Circulation 2018;137(22):2344-56. http://dx.doi.org/10.1161/CIRCULATIONAHA.117.032380.

4. Forouzanfar MH, Liu P, Roth GA, Ng M, Biryukov S, Marczak L, et al. Global burden of hypertension and systolic blood pressure of at least 110 to $115 \mathrm{~mm} \mathrm{Hg}, 1990-2015$. JAMA 2017;317(2):165-82. http://dx.doi.org/10.1001/jama.2016.19043.

5. Padwal RS, Bienek A, McAlister FA, Campbell NR, Outcomes Research Task Force of the Canadian Hypertension Education Program. Epidemiology of hypertension in Canada: an update. Can J Cardiol 2016;32(5):687 - 94. http://dx.doi.org/10.1016/j.cjca.2015.07. 734.

6. World Health Organization. Hypertension care in Thailand: best practices and challenges, 2019. https://apps.who.int/iris/handle/10665/
330488. [2020-9-20].

7. Kontis V, Cobb LK, Mathers CD, Frieden TR, Ezzati M, Danaei G. Three public health interventions could save 94 million lives in 25 years. Circulation 2019;140(9):715 - 25. http://dx.doi.org/10.1161/ CIRCULATIONAHA.118.038160.

8. Farley TA, Dalal MA, Mostashari F, Frieden TR. Deaths preventable in the U.S. by improvements in use of clinical preventive services. Am J Prev Med 2010;38(6):600 - 9. http://dx.doi.org/10.1016/j.amepre. 2010.02.016.

9. Ide N, Ajenikoko A, Steele L, Cohn J, Cohn CJ, Frieden TR, et al. Priority actions to advance population sodium reduction. Nutrients 2020;12(9):2543. http://dx.doi.org/10.3390/nu12092543.

10. Frieden TR, Cobb LK, Leidig RC, Mehta S, Kass D. Reducing premature mortality from cardiovascular and other non-communicable diseases by one third: achieving Sustainable Development Goal indicator 3.4.1. Glob Heart 2020;15(1):50. http://dx.doi.org/10. 5334/gh.531.

11. National Health Commission of the People's Republic of China. Healthy China Action Plan (2019-2030): campaign to prevent disease and promote health. National Health Commission of the People's Republic of China. http://en.nhc.gov.cn/HealthyChinaActionPlan. html. [2020-9-20].

12. Frieden TR, Jaffe MG. Saving 100 million lives by improving global treatment of hypertension and reducing cardiovascular disease risk factors. J Clin Hypertens 2018;20(2):208 - 11. http://dx.doi.org/10. $1111 /$ jch. 13195 .

13. World Health Organization. HEARTS: Technical package for cardiovascular disease management in primary health care. Geneva: World Health Organization; 2016. http://www.who.int/cardiovascular_ diseases/hearts/en.[2020-9-20].

14. The World Bank and World Health Organization. Deepening health reform in China building high-quality and value-based service delivery. New York: The World Bank and World Health Organization, 2019. http://documents1.worldbank.org/curated/en/690791553844042874/ pdf/Building-High-Quality-and-Value-Based-Service-Delivery.pdf. [2020-9-20].

15. Yip W, Fu HQ, Chen AT, Zhai TM, Jian WY, Xu RM, et al. 10 years of health-care reform in China: progress and gaps in Universal Health Coverage. Lancet 2019;394(10204):1192 - 204. http://dx.doi.org/10. 1016/S0140-6736(19)32136-1.

16. He FJ, Brinsden HC, MacGregor GA. Salt reduction in the United Kingdom: a successful experiment in public health. J Hum Hypertens 2014;28(6):345 - 52. http://dx.doi.org/10.1038/jhh.2013.105.

17. Karppanen H, Mervaala E. Sodium intake and hypertension. Prog Cardiovasc Dis 2006;49(2):59 - 75. http://dx.doi.org/10.1016/j.pcad. 2006.07.001.

18. Park HK, Lee Y, Kang BW, Kwon KI, Kim JW, Kwon S, et al. Progress on sodium reduction in South Korea. BMJ Glob Health 2020; 5(5):e002028. http://dx.doi.org/10.1136/bmjgh-2019-002028.

19. Du SF, Wang HJ, Zhang B, Popkin BM. Dietary potassium intake remains low and sodium intake remains high, and most sodium is derived from home food preparation for Chinese adults, 1991-2015 trends. J Nutr 2020;150(5):1230 - 9. http://dx.doi.org/10.1093/jn/ nxz332.

20. Xu AQ, Ma JX, Guo XL, Wang LH, Wu J, Zhang JY, et al. Association of a province-wide intervention with salt intake and hypertension in Shandong Province, China, 2011-2016. JAMA Intern Med 2020; 180(6):877 - 86. http://dx.doi.org/10.1001/jamainternmed.2020.0904.

21. Taillie LS, Reyes M, Colchero MA, Popkin B, Corvalán C. An evaluation of Chile's Law of Food Labeling and Advertising on sugarsweetened beverage purchases from 2015 to 2017: a before-and-after study. PLoS Med 2020;17(2):e1003015. http://dx.doi.org/10.1371/ journal.pmed.1003015.

22. Niebylski ML, Lu T, Campbell NR, Arcand J, Schermel A, Hua DE, et al. Healthy food procurement policies and their impact. Int J Environ Res Public Health 2014;11(3):2608 - 27. http://dx.doi.org/10.3390/ ijerph 110302608.

23. Ma LY, Chen WW, Gao RL, Liu LS, Zhu ML, Wang YJ, et al. China cardiovascular diseases report 2018: an updated summary. J Geriatr Cardiol 2020;17(1):1 - 8. http://dx.doi.org/10.11909/j.issn.1671-5411. 2020.01.001. 